\title{
Arterial remodelling of native human coronary arteries in patients with unstable angina pectoris: a prospective intravascular ultrasound study
}

\author{
M Gyöngyösi, P Yang, A Hassan, F Weidinger, H Domanovits, A Laggner, D Glogar
}

\begin{abstract}
Objective-To investigate the use of intravascular ultrasound (IVUS) in detecting the presence of arterial remodelling in patients with unstable angina.

Design-Prospective case study.

Patients-60 of 95 consecutively admitted patients with unstable angina (41 male, 19 female), mean (SD) age 61.2 (8.1) years. Interventions-Qualitative and quantitative coronary angiography and IVUS.

Main outcome measures-Adaptive or constrictive remodelling (AR, CR) was considered present when the cross sectional area of the external elastic membrane at the lesion site was larger than the proximal cross sectional area or smaller than the distal cross sectional area, respectively.

Results-22 of the 60 patients $(37 \%)$ showed AR and 14 (23\%) showed CR. No remodelling was seen in 24 patients (group NR). The plaque contained more thrombus and plaque rupture in group AR than in groups CR and NR (thrombus: $91 \% v$ $50 \%$ and $67 \%$, respectively, $p=0.023$; rupture: $73 \% v 29 \%$ and $42 \%, p=0.020)$. AR was associated with a larger plaque cross sectional area (12.6 (SD 4.6) $\mathrm{mm}^{2} v 10.8$ (6.3) and $\left.9.2(3.7) \mathrm{mm}^{2}, \mathrm{p}=0.001\right)$ and larger external elastic membrane cross sectional area $\left(16.5(5.8) \mathrm{mm}^{2} v 13.2(5.2)\right.$ and $14.4(3.6) \mathrm{mm}^{2}, \mathrm{p}=0.01$ in group $\mathrm{AR} v$ groups $\mathrm{CR}$ and NR, respectively), while the plaque burden was larger in groups AR (74.9 (9.1)\%) and CR (72.4 (16.6)\%) than in group NR (66.2 (18.1)\%, $\mathrm{p}=0.005$ ).
\end{abstract}

Conclusions-IVUS is capable of detect-

2nd Department of

Internal Medicine,

Division of Cardiology,

University of Vienna,

Währinger Gürtel

18-20, A-1090 Vienna,

Austria

M Gyöngyösi

P Yang

A Hassan

$\mathrm{F}$ Weidinger

D Glogar

Department of Emergency Medicine, University of Vienna H Domanovits

A Laggner

Correspondence to:

Dr Gyöngyösi.

Accepted for publication 21 December 1998

of target lesions and its relation to plaque morphology in unstable angina.

(Heart 1999;82:68-74)

Keywords: unstable angina; intravascular ultrasound; arterial remodelling; angiography

Although the pathogenesis of acute coronary syndromes has not been fully elucidated, plaque disruption and thrombus formation are considered to be crucial events in the development of unstable angina and acute myocardial infarction. ${ }^{1-10}$ Intravascular ultrasound (IVUS) allows direct visualisation of the arterial wall from inside and provides new information on the atherosclerotic plaque composition and its relation to clinical syndromes. ${ }^{10-13}$ Some inves- tigators have reported that culprit lesions in patients with unstable angina are predominantly occupied by soft plaques, while hard plaques are more common in patients with chronic stable coronary disease. ${ }^{14} 15$

Compensatory enlargement of human coronary arteries in response to progressive atherosclerosis was first observed by Glagov et al in 1987 in a histopathological study of human necropsy specimens of left main coronary arteries. ${ }^{16}$ Vessel enlargement (adaptive remodelling) is a compensatory mechanism in response to atherosclerotic progression, to maintain or even enlarge the lumen size. ${ }^{17-22}$ Although such compensatory enlargement seems to be a uniform mechanism in native coronary arteries, Pasterkamp et al found that paradoxical arterial wall shrinkage may contribute to the luminal narrowing of human atherosclerotic femoral arteries. ${ }^{23}$ The presence of shrinkage as constrictive remodelling of the native atherosclerotic human coronary arteries has been confirmed by several investigators. ${ }^{2124-26}$ In a small cohort of patients with unstable coronary syndrome, a low incidence of adaptive remodelling was found; it is thought that the rapid increase in plaque mass does not allow time for adaptive remodelling. ${ }^{25}$ However, no prospective studies have been carried out with larger patient cohorts to investigate the remodelling process of culprit lesions evoking unstable coronary syndrome.

Accordingly, in this prospective study IVUS was employed to investigate arterial remodelling of human native coronary arteries in patients with unstable coronary syndromes; to determine the relation between adaptive or constrictive remodelling and the plaque morphology; and to evaluate the possible correlation between the presence or absence of arterial remodelling and risk factors for coronary atherosclerosis.

\section{Methods}

PATIENT POPULATION

Between September 1995 and March 1997, 95 consecutive patients with unstable angina (70 men and 25 women, mean (SD) age 62 (12) years) were admitted to the department of emergency medicine and enrolled in a prospective study with the following inclusion criteria: new onset of severe or accelerated angina $(<2$ months of duration) (25 patients); angina at rest (52 patients); angina within two weeks of acute myocardial infarction (18 patients) (Braunwald class IA, IIA, IIIA, IB, IIB, IIIB, or IIIC). ${ }^{27}$ The Braunwald categories of unstable 
angina were grouped according to the scoring system established by Ahmed et al. ${ }^{6}$ Three subgroups with increasing severity of symptoms were created: low (IA, IIA, IIIA, IB), medium (IIB, IIIB), and high (IIIC). Subjects were excluded from the study if any of the following exclusion criteria were present: acute myocardial infarction; previous coronary intervention at the site of the target lesion; the presence of other serious illness; or an inability or unwillingness to comply with the protocol.

In all the patients, an ECG during angina revealed new ST segment depressions or elevations in at least two leads. The initial creatine kinase level was normal. All patients underwent the same antianginal treatment-intravenous glyceryl trinitrate and heparin. Data relating to coronary risk factors were recorded, including diabetes mellitus (drug dependent only), hypertension (drug treated only), and hypercholesterolaemia (drug treated or serum cholesterol $\geqslant 6.2 \mathrm{mmol} / \mathrm{l}$ ), and smoking habits.

The study protocol was approved by the local ethics committee, and written informed consent was obtained from all patients.

CORONARY ANGIOGRAPHY AND IVUS PROCEDURES After administration of 150 to $200 \mu \mathrm{g}$ of intracoronary glyceryl trinitrate, all patients underwent selective coronary angiography. Baseline angiograms were recorded in at least two projections. After completion of coronary angiography, culprit lesions were localised and IVUS imaging was performed. Intracoronary glyceryl trinitrate $(200 \mu \mathrm{g})$ was given before intracoronary ultrasound imaging to prevent vasospasm, and the imaging catheter was introduced through an $8 \mathrm{~F}$ guiding catheter over a guide wire.

Intracoronary ultrasound images were obtained with $2.9 \mathrm{~F}$ or $3.2 \mathrm{~F}$ mechanical imaging catheters (CVIS, Sunnyvale, California, USA) or $3.0 \mathrm{~F}$ electronic imaging catheters (Endosonics, California, USA). The IVUS catheter was advanced distally and subsequently withdrawn manually. Correct assessment of the IVUS catheter position and the site of the culprit lesion was achieved by fluoroscopic control or angiographic documentation of the tip of the catheter, or both. All IVUS images were obtained at 30 frames/second and recorded on super VHS videotapes for subsequent offline analysis. Selected images from the videotape were digitised and stored in computer based patient data files.

\section{CULPRIT LESION IDENTIFICATION}

The culprit lesion was identified according to the ECG pattern and angiographic vessel morphology. In patients with single vessel disease, the diseased artery was considered to be the ischaemia related artery. In patients with multivessel disease, the localisation of the ECG changes during anginal episodes, the angiographic appearance of thrombus, and the most severe stenosis or complex lesion morphology were used to identify the ischaemia related artery. If, for any reason, no clear culprit lesion was detectable (normal or nearly normal angiographic and IVUS findings, severe multi- vessel disease, and so on), the case was treated as a drop out.

ANGIOGRAPHIC ANALYSIS

Cineangiograms were analysed by two experienced observers blind to the ultrasound results in two different sessions, using a computer assisted quantitative coronary arteriographic edge detection algorithm (Cardiovascular Measurement System, Medis, the Netherlands). Minimum lumen and reference diameters, lengths of stenoses, per cent diameter stenoses, and per cent area stenoses were measured at end diastolic frames to minimise the variation caused by the cardiac motion and to maximise the contrast filling of the coronary vessel. The TIMI (thrombolysis in myocardial infarction study) flow, culprit lesion morphology, and the presence of thrombi or filling defects, native vessel dissections, calcification, and collateral vessels were analysed visually. ${ }^{28}$ Concentric angiographic lesions with a smooth border were considered to be simple angiographic lesions, while eccentric lesions with a narrow neck, overhanging edges, or irregular borders were regarded as complex lesions. ${ }^{28}$ Lesion length was measured as the distance $(\mathrm{mm})$ from the proximal shoulder to the distal shoulder in the projection with the least foreshortening. ${ }^{29}$

IVUS ANALYSES

IVUS images were analysed in offline mode by two experienced observers with a computer assisted IVUS analysis system (TapeMeasure, Indec Systems Inc, California, USA).

\section{Qualitative IVUS analysis}

Consensus between the two independent observers was reached in all cases with regard to the qualitative features of the culprit lesion. The atherosclerotic plaque encompassing the culprit lesion was characterised as follows.

Plaque composition - Soft (fibromuscular, lipids, or both) or fibrocalcific (fibrotic but also calcified) lesions. Tissue less dense than the reference adventitia was classified as soft. Plaque tissue producing echoes that were as bright as or brighter than the reference adventitia, with or without acoustic shadowing was classified as fibrocalcific. Bright echoes with acoustic shadowing were considered to represent calcification.

Plaque eccentricity-Plaque was considered eccentric if the ratio of plaque thickness on opposite sides of the lumen was $<0.5$ or if there was an arc of disease-free vessel wall.

Plaque disruptions or thrombi-These were identified visually. Plaque disruption was defined as an abrupt, focal, superficial break in the linear continuity of the plaque that extended in a radial direction only. Vascular thrombi were considered to exist when speckled echoes softer than the dense atheroma echo signal within soft plaque were seen.

\section{Quantitative IVUS analysis}

The site of the minimum lumen cross sectional area was identified by carefully scrolling the tape forwards and backwards. For each lesion 
site, the lumen area and external elastic membrane area (defined as the area encompassed by the adventitia) were measured at the point of maximum lumen narrowing and in adjacent proximally and distally located segments (that is, reference sites). The reference segments were selected on the basis of the segment morphology determined by angiography (minimal or no lumen narrowing, no major side branch originating between the lesion site and the reference site) or IVUS (normal or less diseased vessel segment with $<50 \%$ area stenoses and without active plaques), or both. The location of the culprit lesion and the proximal and distal reference sites was determined after consensus by two observers. Quantitative analyses were performed by two independent observers. For further comparison, the measurements of the three observers were averaged. For each lesion the lumen cross sectional area (area within the lumen-intima border $\left(\mathrm{mm}^{2}\right)$ ) and external elastic membrane cross sectional area were manually traced. Plaque cross sectional area was measured as intima + media area, calculated as external elastic membrane cross sectional area - lumen cross sectional area $\left(\mathrm{mm}^{2}\right)$. Per cent plaque burden was calculated as (plaque cross sectional area/external elastic membrane cross sectional area) $\times 100[\%]$.

\section{Assessment of reproducibility}

Cross sectional measurements on 19 different coronary artery lesions were analysed by two observers in separate sessions, and the interobserver variability was calculated on the basis of IVUS images measured by these observers. For determination of the intraobserver variability, 24 lesions were measured five times by one observer. The different analyses included the error involved in repeatedly selecting the same image slice and the error involved in repeatedly performing the cross sectional measurements.

The coefficient of correlation of the interobserver variability was $r=0.956(\mathrm{p}<0.001)$.

The intraobserver variability and the reproducibility of IVUS measurements were determined by using one way analysis of variance with repeated measurements. The methodological error was calculated from the standard error of the estimate of the analysis of variance.

The coefficient of variation of the repeated measurements of the lumen and external elastic membrane diameter was $3 \%$, while that of the lumen and external elastic membrane cross sectional area was $1.7 \%$. The methodological error of the measurement of the lumen and external elastic membrane diameter was 0.19 $\mathrm{mm}$, and that for the lumen and external elastic membrane cross sectional area, $0.38 \mathrm{~mm}^{2}$.

\section{Arterial remodelling}

Adaptive remodelling (compensatory vessel enlargement) was assumed to be present when the external elastic membrane cross sectional area at the lesion site was larger than the proximal reference external elastic membrane cross sectional area.

Constrictive remodelling (coronary shrinkage) was considered to be present when the external elastic membrane cross sectional area
95 consecutive patients with unstable angina pectoris and coronary angiography

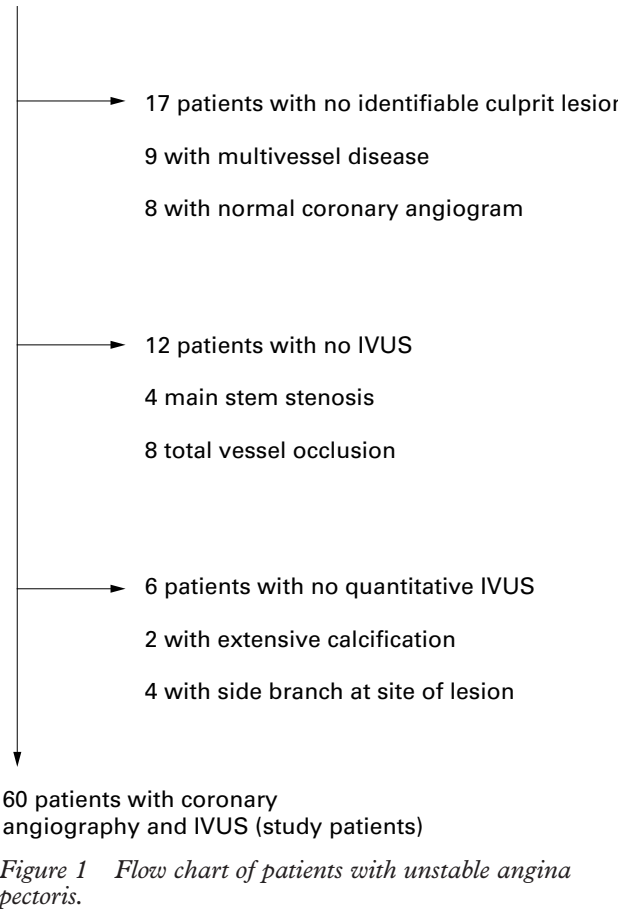

at the lesion site was smaller than that at the distal reference site.

\section{STATISTICS}

All data are expressed as means (SD) for continuous variables. Qualitative data are presented as frequencies. Comparisons between groups were made with the Student's $t$ test, or with analysis of variance for differences in means, or with the $\chi^{2}$ test for categorical variables. Regression analysis was used to assess the interobserver variability, and one way analysis of variance with repeated measurement was applied to assess the reproducibility of repeated measurements for determination of the intraobserver variability. Differences were considered significant when $\mathrm{p}<0.05$.

\section{Results}

Qualitative and quantitative data on the culprit lesion and the proximal and distal reference segments could be determined in 60 patients (41 men and 19 women, mean age 61 (8) years). Culprit lesions could not be identified in 17 of the 95 patients because of multivessel disease (nine patients) or a normal coronary angiogram (eight patients). In these eight patients, even IVUS did not detect any possible pathological substrate in any of the three coronary arteries. These eight patients were in class IA, IIA, or IIIA of the Braunwald classification of unstable angina. In another 12 patients, IVUS could not be performed owing to a severe main stem stenosis (four patients) or total vessel occlusion (eight patients). Furthermore, in six patients quantitative IVUS measurements were not possible because of extensive calcification (calcification occupying more than $90^{\circ}$ of the vessel wall) (two patients), or the presence of a side branch at the 


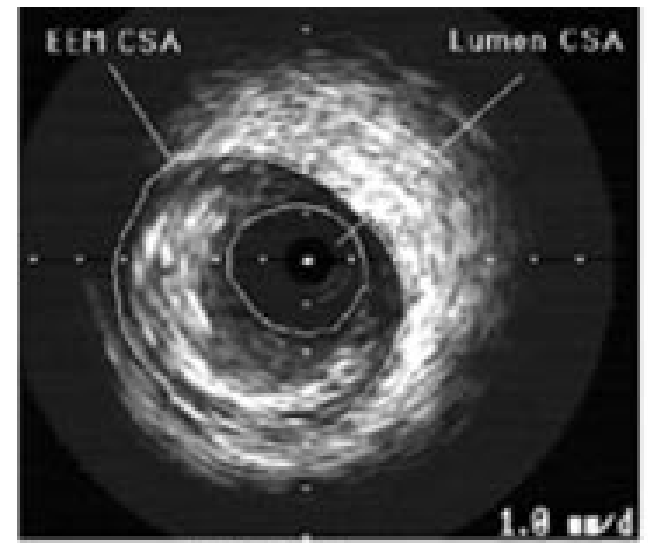

Culprit lesion

vessel ares: $17.61 \mathrm{~mm}^{2}$ lumen area: $4.23 \mathrm{~mm}^{2}$ plasque burden: $76 \%$

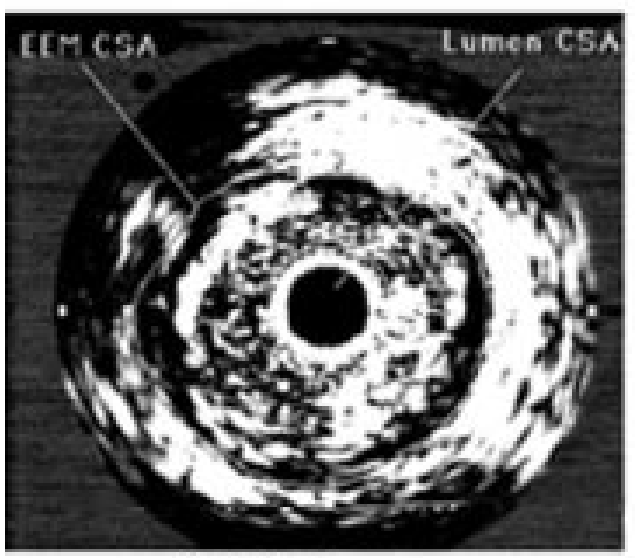

Culprit lesion

vessel area: $17.34 \mathrm{~mm}^{2}$

lumen area: $2.77 \mathrm{~mm}^{2}$ plaque burden: $84 \%$

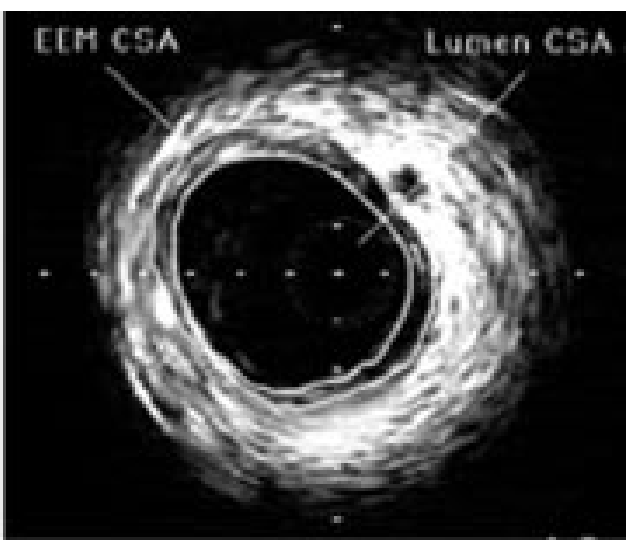

Proximal reterence segment vessel area: $16.04 \mathrm{~mm}^{2}$ lumen areac $11.88 \mathrm{~mm}^{2}$ plaque burden: $21 \%$

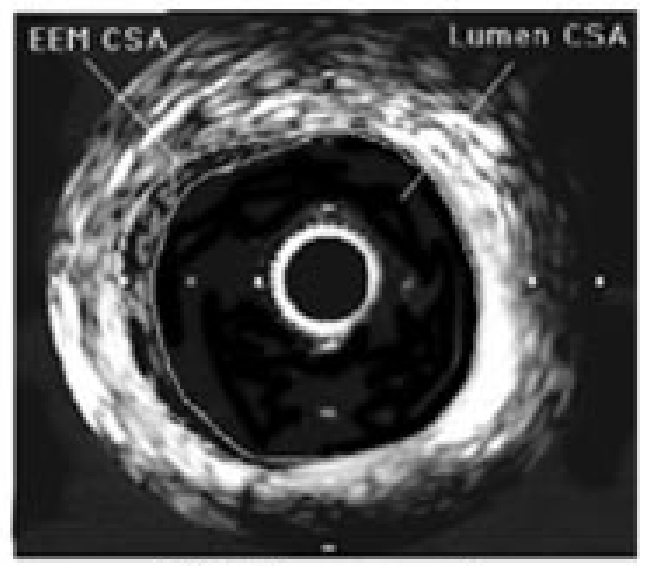

Distal reference segment vessel area: $18.94 \mathrm{~mm}^{2}$ lumen arsa: $16.88 \mathrm{~mm}^{2}$ plaque burden: $11 \%$

Figure 2 Adaptive remodelling (compensatory vessel enlargement; upper panels) and constrictive remodelling (shrinkage; lower panels) of the native coronary artery in a patient with unstable angina. CSA, cross sectional area; EEM, external elastic membrane.

site of the culprit lesion (four patients), which did not allow delineation of the vessel wall contour (fig 1). Thus the present results are the data on the remaining 60 patients.

In three patients, transient coronary spasm occurred after the introduction of the IVUS catheter, but after withdrawal of the catheter and administration of a further dose of intra-

Table 1 Clinical data for patients with adaptive remodelling of the culprit lesion (group $A R$ ), patients with constrictive remodelling (group CR), and patients without remodelling (group NR)

\begin{tabular}{lclll}
\hline & $\begin{array}{l}\text { Group AR } \\
(n=22)\end{array}$ & $\begin{array}{l}\text { Group CR } \\
(n=14)\end{array}$ & $\begin{array}{l}\text { Group } N R \\
(n=24)\end{array}$ & $\begin{array}{l}\text { Total } \\
(n=60)\end{array}$ \\
\hline $\begin{array}{l}\text { Age (years) (mean (SD)) } \\
\text { Male }\end{array}$ & $56.5(14.0)$ & $65.4(8.1)^{\star}$ & $60.9(9.8)$ & $61.2(8.1)$ \\
Angina score & $16(73)$ & $8(57)$ & $17(71)$ & $41(68)$ \\
$\quad$ Low & $5(23)$ & $0(0)$ & $8(33)$ & $13(22)$ \\
$\quad$ Medium & $13(59)$ & $6(43)$ & $10(42)$ & $29(48)$ \\
$\quad$ High & $4(18)$ & $8(57) \dagger$ & $6(25)$ & $18(30)$ \\
Risk factors & $4(18)$ & $5(36)$ & $4(17)$ & $13(22)$ \\
$\quad \begin{array}{l}\text { Diabetes } \\
\text { Hypertension }\end{array}$ & $8(36)$ & $10(71) \ddagger$ & $12(50)$ & $30(50)$ \\
$\quad$ Hypercholesterolaemia & $13(59)$ & $8(57)$ & $16(67)$ & $37(62)$ \\
$\quad$ Smoking & $13(59)$ & $7(50)$ & $13(54)$ & $33(55)$ \\
\hline
\end{tabular}

Values are $\mathrm{n}(\%)$ except where stated.

${ }^{\star} p=0.045, \dagger p=0.036, \neq p=0.04$ for comparisons between group CR and groups AR/NR. coronary glyceryl trinitrate, the IVUS procedure could be performed. Before the IVUS procedure, an intracoronary bolus injection followed by intravenous infusion of abciximab (ReoPro; Eli Lilly) was given to two patients because of extensive intracoronary thrombus on angiography.

ADAPTIVE AND CONSTRICTIVE REMODELLING Clinical results

Twenty two of the 60 patients $(37 \%)$ showed adaptive remodelling on IVUS, while constrictive remodelling of the culprit lesion was observed in 14 patients (23\%) (fig 2). Table 1 summarises the clinical data on the patients with adaptive (group AR) and constrictive remodelling (group CR) and the patients with no remodelling (group NR). Patients with constrictive remodelling were significantly older (65.4 (8.1) years, 95\% confidence interval 60.7 to 70.0) than the patients in group AR (56.5 (14) years, 50.1 to 60.9$)$ and group NR (60.9 (9.8) years, 56.8 to 65.1 ), $\mathrm{p}=0.045$, and had a significantly more severe angina score (table 1 ). 
Table 2 Qualitative and quantitative coronary angiography data on patients with adaptive remodelling of the culprit lesion (group AR), patients with constrictive remodelling (group CR), and patients without remodelling (group NR)

\begin{tabular}{lcccc}
\hline & $\begin{array}{l}\text { Group AR } \\
(n=22)\end{array}$ & $\begin{array}{l}\text { Group CR } \\
(n=14)\end{array}$ & $\begin{array}{l}\text { Group NR } \\
(n=24)\end{array}$ & $\begin{array}{l}\text { Total } \\
(n=60)\end{array}$ \\
\hline $\begin{array}{l}\text { Qualitative angiographic data (n (\%)) } \\
\text { One vessel disease }\end{array}$ & $11(50)$ & $5(36)$ & $17(71)$ & $30(50)$ \\
Simple lesion & $11(50)$ & $6(43)$ & $10(42)$ & $27(45)$ \\
Complex lesion & $11(50)$ & $8(57)$ & $14(58)$ & $33(55)$ \\
Proximal lesion & $12(55)$ & $5(36)$ & $15(63)$ & $32(53)$ \\
Quantitative angiographic data (mean (SD)) & & & \\
Minimal lumen diameter (mm) & $1.38(0.67)$ & $1.62(0.5)$ & $1.68(0.66)$ & $1.57(0.64)$ \\
Reference diameter (mm) & $2.65(0.77)$ & $3.06(0.58)$ & $2.78(0.68)$ & $2.90(0.80)$ \\
Diameter stenosis & $59.7(22.4)$ & $56.5(11.9)$ & $48.1(19.9)$ & $54.3(20.0)$ \\
Area stenosis & $78.8(18.1)$ & $79.8(9.9)$ & $69.2(19.5)$ & $75.2(17.7)$ \\
Stenosis length & $7.25(4.31)$ & $10.2(3.16)^{\star}$ & $8.19(4.62)$ & $8.31(4.32)$ \\
\hline
\end{tabular}

${ }^{\star} \mathrm{p}=0.037$ for comparisons between group $\mathrm{CR}$ and groups $\mathrm{AR} / \mathrm{NR}$.

Table 3 Qualitative intravascular ultrasound data on patients with adaptive remodelling of the culprit lesion (group AR), patients with constrictive remodelling (group CR), and patients without remodelling (group NR)

\begin{tabular}{lclll}
\hline & $\begin{array}{l}\text { Group AR } \\
(n=22)\end{array}$ & $\begin{array}{l}\text { Group CR } \\
(n=14)\end{array}$ & $\begin{array}{l}\text { Group } N R \\
(n=24)\end{array}$ & $\begin{array}{l}\text { Total } \\
(n=60)\end{array}$ \\
\hline $\begin{array}{l}\text { Plaque eccentricity } \\
\text { Concentric lesion }\end{array}$ & $6(25)$ & $7(50)$ & $9(38)$ & $22(37)$ \\
$\begin{array}{l}\text { Eccentric lesion } \\
\text { Plaque composition }\end{array}$ & $16(75)$ & $7\{50)$ & $15(62)$ & $38(63)$ \\
$\begin{array}{l}\text { Soft plaque } \\
\text { Fibrocalcific plaque }\end{array}$ & $13(59)$ & $8(57)$ & $14(58)$ & $35(58)$ \\
Thrombus & $9(41)$ & $6(43)$ & $10(42)$ & $25(42)$ \\
$\begin{array}{l}\text { Disruption } \\
\text { Calcification }\end{array}$ & $20(91)^{\star}$ & $7(50)$ & $16(67)$ & $43(72)$ \\
\hline
\end{tabular}

Values are $\mathrm{n}(\%)$.

${ }^{\star} \mathrm{p}=0.023, \mathrm{tp}=0.020$ for comparisons between group AR and groups CR/NR

Constrictive remodelling tended to be more common in patients with hypertension $33 \% v$ $13 \%, p=0.067$ in patients with or without hypertension). No other correlation was found between coronary risk factors and remodelling.

Qualitative and quantitative angiographic results Coronary angiography indicated a simple lesion in 27 patients (45\%). TIMI flow 3 was seen in 44 patients $(73 \%)$, and a filling defect was observed within the culprit lesion in 20 patients $(33 \%)$. Collateral circulation supplied the diseased vessel in eight patients (13\%). Culprit lesion dissection was identified in five patients $(8 \%)$ and calcification in four $(7 \%)$. Thirty patients $(50 \%)$ had single vessel disease, 21 patients $(35 \%)$ had two vessel disease, and nine patients $(15 \%)$ had three vessel disease.

Table 4 Quantitative intravascular ultrasound data on patients with adaptive remodelling of the culprit lesion (group AR), patients with constrictive remodelling (group CR), and patients without remodelling (group NR)

\begin{tabular}{|c|c|c|c|c|}
\hline & $\begin{array}{l}\text { Group } A R \\
(n=22)\end{array}$ & $\begin{array}{l}\text { Group CR } \\
(n=14)\end{array}$ & $\begin{array}{l}\text { Group NR } \\
(n=24)\end{array}$ & Total $(n=60)$ \\
\hline \multicolumn{5}{|l|}{ Culprit lesion } \\
\hline Lumen CSA $\left(\mathrm{mm}^{2}\right)$ & $4.0(2.0)$ & $3.3(1.3)$ & $4.6(2.3)$ & $4.01(2.1)$ \\
\hline Plaque CSA $\left(\mathrm{mm}^{2}\right)$ & $12.6(4.6)^{\star}$ & $10.8(6.3)$ & $9.2(3.7)$ & $10.8(4.9)$ \\
\hline $\mathrm{EEM} \mathrm{CSA}\left(\mathrm{mm}^{2}\right)$ & $16.5(5.8)+$ & $13.2(5.2)$ & $14.4(3.6)$ & $14.9(5.0)$ \\
\hline Plaque burden (\%) & $74.9(9.1)$ & $72.4(16.6)$ & $66.2(18.1) \ddagger$ & $70.1(15.3)$ \\
\hline \multicolumn{5}{|l|}{ Proximal reference segment } \\
\hline Lumen CSA $\left(\mathrm{mm}^{2}\right)$ & $8.5(2.4)$ & $10.7(3.2)$ & $9.4(2.0)$ & $9.5(2.8)$ \\
\hline Plaque CSA $\left(\mathrm{mm}^{2}\right)$ & $5.4(4.1)$ & $6.5(2.2)$ & $6.1(3.8)$ & $6.0(3.5)$ \\
\hline $\operatorname{EEM~CSA~}\left(\mathrm{mm}^{2}\right)$ & $13.9(5.0)$ & $15.8(5.2)$ & $15.5(4.5)$ & $15.4(4.9)$ \\
\hline Plaque burden (\%) & $35.8(14.4)$ & $37.1(8.1)$ & $36.9(14.1)$ & $36.7(9.3)$ \\
\hline \multicolumn{5}{|l|}{ Distal reference segment } \\
\hline Lumen CSA $\left(\mathrm{mm}^{2}\right)$ & $8.2(3.5)$ & $8.8(3.2)$ & $7.4(2.4)$ & $8.1(3.1)$ \\
\hline Plaque CSA $\left(\mathrm{mm}^{2}\right)$ & $4.2(2.5)$ & $5.2(3.2)$ & $3.6(2.4)$ & $4.3(2.8)$ \\
\hline $\operatorname{EEM~CSA~}\left(\mathrm{mm}^{2}\right)$ & $12.3(5.1)$ & $13.5(4.8)$ & $11.7(4.0)$ & $12.4(4.2)$ \\
\hline Plaque burden (\%) & $33.9(11.7)$ & $35.3(13.3)$ & $28.7(12.5)$ & $31.2(12.2)$ \\
\hline
\end{tabular}

Values are mean (SD).

${ }^{\star} \mathrm{p}=0.001, \mathrm{tp}=0.01$ for comparisons between group $\mathrm{AR}$ and groups $\mathrm{CR} / \mathrm{NR}$.

$\neq \mathrm{p}=0.005$ for comparisons between group $\mathrm{NR}$ and groups $\mathrm{AR} / \mathrm{CR}$.

CSA, cross sectional area; EEM, external elastic membrane.
There was no statistical difference in qualitative angiographic morphology of the culprit lesion in the different vessels and in relation with remodelling.

Table 2 summarises the qualitative and quantitative angiographic data. There was no significant difference between the three groups in minimum luminal diameter, reference diameter, or diameter or area stenosis of the culprit lesion. The average stenosis length was greater in group CR (10.2 (3.16) $\mathrm{mm}, 95 \%$ confidence interval 8.38 to 12.02$)$ than in group $\mathrm{AR}(7.25$ (4.31), 5.29 to 9.2$)$ and group NR (8.19 (4.62), 6.25 to 10.14$) ; \mathrm{p}=0.037$.

\section{Qualitative IVUS data}

Intracoronary thrombi and plaque disruption were more common in patients with adaptive remodelling than in those without (thrombus: $91 \% v 50 \%$ and $67 \%, \mathrm{p}=0.023$; rupture: $73 \%$ $v 29 \%$ and $25 \%, \mathrm{p}=0.020$, in group $\mathrm{AR} v$ groups CR and NR). There were no other differences between the patient groups with regard to the plaque eccentricity and composition (table 3).

\section{Quantitative IVUS results}

Adaptive remodelling was associated with a larger plaque cross sectional area (12.6 (4.6) $\mathrm{mm}^{2}, 95 \%$ confidence interval 10.46 to 14.66 , v $10.8(6.3) \mathrm{mm}^{2}, 7.14$ to 14.46 , and 9.2 (3.7) $\mathrm{mm}^{2}, 7.65$ to 10.79 , in group AR $v$ groups CR and NR, respectively; $p=0.001$ ) and a larger external elastic membrane cross sectional area (16.5 (5.8) $\mathrm{mm}^{2}, 13.9$ to $19.13, v 13.2(5.2)$ $\mathrm{mm}^{2}, 10.02$ to 16.29 , and 14.4 (3.6) $\mathrm{mm}^{2}$, 12.87 to 15.95 , in group AR $v$ groups CR and $\mathrm{NR}$, respectively; $\mathrm{p}=0.01$ ), while the plaque burden was higher in groups AR (74.9 (9.1)\%, 70.8 to 79.0$)$ and CR $(72.4(16.6) \%, 62.8$ to 81.9) than in group NR $(66.2(18.1) \%, 58.5$ to 73.8; $\mathrm{p}=0.005)$ (table 4).

The average minimum lumen, plaque, and vessel cross sectional areas of the proximal and distal reference sites were not different in the different remodelling processes.

\section{Discussion}

In this prospective IVUS study we have demonstrated different arterial remodelling processes in native coronary arteries in patients with unstable angina.

\section{IVUS VARIATIONS IN DIFFERENT REMODELLING} PROCESSES

We found that $37 \%$ of the patients with unstable angina had adaptive remodelling and $23 \%$ had constrictive remodelling. Tauth et al found that $35 \%$ of the target lesions showed adaptive remodelling while $34 \%$ of the lesions showed constrictive remodelling in a mixed patient group with stable and unstable angina..$^{30}$ Mintz et al reported inadequate remodelling in at least $15 \%$ of chronic focal new coronary arterial stenoses in patients with stable angina. ${ }^{26} \mathrm{Nishi-}$ oka et al found that vessel enlargement failure occurred in $46 \%$ of target lesions. ${ }^{24}$ The differences between the our results and those of other investigators can be explained in terms of the different patient populations - in our study 
we included only patients with unstable angina, because of growing evidence that remodelling of the coronary arteries related to unstable coronary syndromes is different from that in stable angina, just as the mechanisms of lesion progression are considered to be different as well. $^{28} 31$

Qualitative IVUS revealed a high incidence of thrombi and plaque disruption in the patients as a whole. This finding is in agreement with the angiographic and pathological evidence that lesion progression in acute coronary syndromes follows recurrent minor fissuring of the most fatty atheromatous plaques, with subsequent thrombus formation. $^{2} 28313233$ Moreover, presence of thrombi and plaque disruption was significantly more common in patients with adaptive remodelling than in patients with constrictive remodelling or without any remodelling. It may be hypothesised that vasoactive substances released by the intracoronary thrombi and the disrupted atherosclerotic plaques contribute to more pronounced vessel dilatation in patients with adaptive remodelling. However, if this pathophysiological concept were the only explanation, it would remain completely unclear why adaptive remodelling did not develop in the other patients with unstable angina associated with intracoronary thrombi and plaque disruption.

Another theory suggests that atrophy and thinning of the media induced by atherosclerosis could be responsible for vessel dilatation during remodelling, by weakening the arterial wall. This theory could be confirmed by calculation of the media thickness using additional measurements of the internal elastic membrane area (the difference between the external elastic membrane area and the internal elastic membrane area). Unfortunately, IVUS is not suitable for precise delineation of the internal elastic membrane area in most cases; therefore these measurements were not included in our study. $^{34}$

ARTERIAL REMODELLING AND CLINICAL DATA The patients with constrictive remodelling were significantly older and had a more severe angina score than the patients in the other groups. This finding correlates with the published reports that adaptive remodelling is an early process initiating the maintenance of the coronary blood flow through the narrowed artery, while constrictive remodelling is an advanced process during the course of coronary artery disease and occurs more often after a mechanical or chemical injury to the coronary artery. ${ }^{21232435-39}$ The process leading to constrictive remodelling in patients with unstable angina, including the possibly longer duration of clinically silent coronary artery disease, remains speculative.

REMODELLING AND ANGIOGRAPHIC DATA

Qualitative coronary angiography did not reveal any differences between the different remodelling processes. Among the quantitative angiographic variables, only the stenosis length was significantly greater in patients with constrictive remodelling. Insignificantly smaller lumen and vessel diameters were found in patients with adaptive remodelling on quantitative angiography, in contrast with the significantly greater lumen and external elastic membrane cross sectional areas measured by IVUS. Angiography is lumenography, in contrast to IVUS, and introduces multiple pitfalls for the assessment of arterial remodelling:

- The reference diameter at the site of the target lesion is only a lumen diameter calculated by interpolatating from the lumen diameters of the proximal and distal segments adjacent to the stenosis, and is therefore not a true measure of vessel diameter.

- Lumenography (by definition) cannot depict the enlargement or shrinkage of a vessel.

- Angiographic measurements of the lumen diameters of eccentric plaques (typically present in unstable angina) may result in failure to detect significant variations in vessel calibre. In our study, the differences between the lumen and reference diameters in the different groups were not significant. It seems that IVUS is more suitable for determining lesion characteristics and remodelling processes than qualitative and quantitative coronary angiography.

METHODS OF ASSESSING THE DIFFERENT TYPES OF REMODELLING

The remodelling process may be assessed in different ways. In a serial study with quantitative coronary angiography or IVUS, the development of remodelling (adaptive or constrictive) may be determined in a given patient by serial arterial analyses. As the time course of the arterial wall changes was unknown in our study, we defined adaptive remodelling as an enlargement of the vessel cross sectional area in comparison with a proximal reference site, and constrictive remodelling as a culprit lesion vessel shrinkage in comparison with a distal reference site, in accordance with published reports. ${ }^{1620243640}$

LIMITATIONS OF OUR STUDY

Our findings are based on the observation of 60 primary coronary lesions that excluded ostial lesions and also coronary lesions with severe calcification. Therefore, they might not be applicable to heavily calcified, restenotic and ostial lesions.

The number of the patients in the different groups was relatively small; however, this number was derived from a reasonable sample size of 95 consecutively recruited patients.

For determining the IVUS features in patients with unstable coronary syndromes, we used only a "snapshot view" of the culprit lesion.

Different degrees of vascular tone (spasm from the catheter at the lesion site, or vasodilatation because of glyceryl trinitrate) could produce an artificial narrowing or enlargement of the lumen and vessel area. However, all patients received the same basic antianginal treatment before and during the interventional procedure. If coronary spasm did occur, the IVUS was repeated after withdrawal of the 
catheter and a repeated dose of glyceryl trinitrate, and the last record was used for quantitative analysis.

Long term results are needed to determine the predictive value of the presence or absence of adaptive or constrictive remodelling in patients with unstable angina. We have started to follow up our patients both clinically and angiographically with IVUS, but this follow up study remains to be completed.

\section{CONCLUSIONS}

The clinical conclusions of our findings are as follows. Different arterial responses to luminal narrowing, both adaptive remodelling and constrictive remodelling, were found in patients with unstable angina. Patients with adaptive remodelling showed more thrombi and plaque disruption, and larger plaque and vessel cross sectional areas. Patients with constrictive remodelling were significantly older and had a higher angina score. Overall, intravascular ultrasonography is more suitable than angiography for determining target lesion characteristics in patients with unstable angina.

1 Fuster V, Badimon L, Cohen $\mathrm{M}$, et al. Insights into the pathogenesis of acute ischemic syndromes. Circulation 1988;77:1213-20.

2 Fuster V, Stein B, Ambrose JA, et al. Atherosclerotic plaque rupture and thrombosis. Evolving concepts. Circulation 1990;82 (suppl II):II-1147-59.

3 Chesebro JH, Zoldhelyi P, Fuster V. Pathogenesis of thrombosis in unstable angina. Am f Cardiol 1991;68:2-10B.

4 Davies MJ, Thomas AC. Plaque fissuring: the cause of acute myocardial infarction, sudden ischaemic death and crescendo angina. Br Heart $\mathcal{f}$ 1985;53:363-73.

5 Fitzgerald DJ, Roy L, Catella F, et al. Platelet activation in unstable coronary disease. N Engl f Med 1986;315:983-9.

6 Ahmed WH, Bittl JA, Braunwald E. Relation between clinical presentation and angiographic findings in unstable cal presentation and angiographic findings in unstable angina pectoris, and comparison with

7 Sherman CT, Litvack F, Grundfest W, et al. Coronary angioscopy in patients with unstable angina. $N$ Engl f Med 1986;315:913-19.

8 Mizuno K, Satomura K, Miyamoto A, et al. Angioscopic evaluation of coronary-artery thrombi in acute coronary syndromes. N Engl f Med 1992;362:287-91.

9 Falk E. Unstable angina with fatal outcome: dynamic coronary thrombosis leading to infarction and/or sudden death. Autopsy evidence of recurrent mural thrombosis with peripheral embolization culminating in total vascular occlusion. Circulation 1985;71:699-780.

10 Depre C, Wijns W, Robert AM, et al. Pathology of unstable plaque: correlation with the clinical severity of acute coronary syndromes. $₹ \mathrm{Am}$ Coll Cardiol 1997;30:694-702.

11 Nishimura RA, Edwards WD, Warnes CA, et al. Intravascular ultrasound imaging: in vitro validation and pathologic lar ultrasound imaging: in vitro validation and

12 Potkin BJ, Bartorelli AL, Gessert JM, et al. Coronary artery imaging with intravascular high frequency ultrasound. Circulation 1990;81:1575-85.

13 Weidinger F, Schwarzacher S, Bohm G, et al. Direct evaluation of the atherosclerotic arterial wall: potentials and limitations of intravascular ultrasound. $Z$ Kardiol 1992;81:1-8.

14 Hodgson JMB, Reddy KG, Suneja R, et al. Intracoronary ultrasound imaging: correlation of plaque-morphology with angiography, clinical syndromes and procedural results in patients undergoing coronary angioplasty. $\mathcal{f} \mathrm{Am}$ Coll Cardiol 1993;21:35-44.

15 Rasheed Q, Nair R, Sheehan H, et al. Correlation of intracoronary ultrasound plaque characteristics in atheroscle- rotic coronary artery disease patients with clinical variables. Am 7 Cardiol 1994; 73:753-8.

16 Glagov S, Weisenberg E, Zarins CK, et al. Compensatory enlargement of human atherosclerotic coronary arteries. $N$ Engl $\mathscr{F}$ Med 1987;316:1371-5.

17 Gibbons GH, Dzau VJ. The emerging concept of vascular remodeling. $N$ Engl f Med 1994;330:1431-8.

18 Isner JM. Vascular remodeling: Honey, I think I shrunk the artery. Circulation 1994;89:2937-41.

19 McPherson DD, Serna AJ, Hiratzka LF, et al. Coronary arterial remodeling studies by high-frequency epicardial echocardiography: an early compensatory mechanism in patients with obstructive coronary atherosclerosis. $\mathcal{F} \mathrm{Am}$ patients with obstructive coro

$20 \mathrm{Ge} \mathrm{J}$, Erbel R, Zamorano J, et al. Coronary artery remodeling in atherosclerotic disease: an intravascular ultrasonic study in vivo. Coron Artery Dis 1993;4:981-6.

21 Kimura T, Kaburagi S, Tamura T, et al. Remodeling of human coronary arteries undergoing coronary angioplasty or atherectomy. Circulation 1997;96:475-83.

22 Birgelen C, Airiian SG, Mintz GS, et al. Variations of remodeling in response to left main atherosclerosis assessed with intravascular ultrasound in vivo. $\mathrm{Am} \neq \mathrm{f}$ Cardiol 1997;80:1408-13.

23 Pasterkamp G, Wensing PJ, Post MJ, et al. Paradoxical arterial wall shrinkage may contribute to luminal narrowing of human atherosclerotic femoral arteries. Circulation 1995; 91:1444-9.

24 Nishioka T, Luo H, Eigler NL, et al. Contribution of inadequate compensatory enlargement to development of human coronary artery stenosis: an in vivo intravascular ultrasound study. $\mathcal{F}$ Am Coll Cardiol 1996;27:1571-6.

25 Gussenhoven EJ, Geselschap JH, van Lankeren W, et al. Remodeling of atherosclerotic coronary arteries assessed with intravascular ultrasound in vitro. Am $f$ Cardiol 1997;79:699-702.

26 Mintz GS, Kent KM, Pichard AD, et al. Contribution of inadequate arterial remodeling to the development of focal coronary artery stenoses. An intravascular ultrasound study. Circulation 1997;95:1791-8.

27 Braunwald E. Unstable angina: a classification. Circulation 1989;80:410-14.

28 Ambrose JA, Winters SL, Stern A, et al. Angiographic morphology and the pathogenesis of unstable angina pectoris. $\mathcal{F}$ Am Coll Cardiol 1985;5:609-16.

29 Popma JJ, Bashore TD. Qualitative and quantitative angiography. In: Topol $\mathrm{E}$ ed. Textbook of interventional cardiology. Philadelphia: WB Saunders, 1994:1052-68.

30 Tauth J, Pinnow E, Sullebarger T, et al. Predictors of coronary arterial remodeling patterns in patients with myocardial ischemia. Am 7 Cardiol 1997;80:1352-5.

31 Ambrose JA, Winters SL, Arora RR, et al. Angiographic evaluation of coronary artery: Morphology in unstable angina. F Am Coll Cardiol 1986;7:472-8.

32 Kearney PP, Erbel R, Rupprecht HJ, et al. Differences in the morphology of unstable and stable coronary lesions and their impact on the mechanisms of angioplasty. An in vivo study with intravascular ultrasound. Eur Heart f 1996;17: $721-30$

33 De Feyter PJ, Ozaki Y, Baptista J, et al. Ischemia-related lesion characteristics in patients with stable or unstable angina. A study with intracoronary angioscopy and angina. A study with intracoronary

34 Kearney PP, Starkey IR, Sutherland GR. Intracoronary ultrasound: current state of the art. Br Heart $\mathcal{F}$ 1995;73:1625.

35 Mintz GS, Popma JJ, Pichard AD, et al. Arterial remodeling after coronary angioplasty. A serial intravascular ultrasound study. Circulation 1996;94:35-43.

36 Lim TT, Liang DH, Botas J, et al. Role of compensatory enlargement and shrinkage in transplant coronary artery disease Serial intravascular ultrasound study. Circulation 1997;95:855-9.

37 Zarins CK, Weisenberg E, Kolletis G, et al. Differential enlargement of artery segments in response to enlarging enlargement of artery segments in response to en
atherosclerotic plaques. $\mathcal{F}$ Vasc Surg 1988;7:386-94.

38 Shi Y, O'Brien JE, Fard A, et al. Adventitial myofibroblasts contribute to neointimal formation in injured porcine coronary arteries. Circulation 1996;94:1655-64.

39 Glagov S. Intimal hyperplasia, vascular remodeling and the restenosis problem. Circulation 1994;89:2888-91.

40 Silva JA, Escobar A, Collins TJ, et al. Unstable angina. A comparison of angioscopic findings between diabetic and nondiabetic patients. Circulation 1995;92:1731-6. 